\title{
A REVIEW ON THERAPEUTIC POTENTIAL OF HETEROCYCLIC PYRIMIDINE DERIVATIVES AS POTENT ANTIVIRAL AGENTS
}

\author{
SAGMA EG*, BASKAR LAKSHMANAN
}

Department of Pharmaceutical Chemistry, Grace College of Pharmacy, Palakkad, Kerala, India. Email: sagmaeg123@gmail.com

Received: 12 March 2020, Revised and Accepted: 20 April 2020

\begin{abstract}
Pyrimidine nucleus exhibited remarkable pharmacological activities. The review of an article indicates that the compounds having pyrimidine nucleus have a wide range of therapeutic uses that include antiviral, anti-inflammatory, antibacterial, anticancer, antiviral, anti-HIV, antihypertensive, sedatives and hypnotics, anticonvulsant, and antihistaminic. This review article is intended to describe the antiviral activity of a compound containing the pyrimidine nucleus. The chemistry of pyrimidine is a thriving field for the study of their pharmacological uses. Numerous methods for the synthesis of pyrimidine as also their diverse reactions offer enormous scope in the field of medicinal chemistry. The review article aims to reveal the work reported on the antiviral synthetic pyrimidine compound and the chemistry and biological activities of pyrimidine during the past few decades. During this review article, we are mainly focusing the viral activities in different derivatives of pyrimidine nucleus. Therefore, we are going to discuss some important issues such as the good ideas to resist our increasing viral disease and the importance of a pyrimidine nucleus in the viral drugs. Hence, these are the main things we are going to discuss in this article.
\end{abstract}

Keywords: Pyrimidine, Anti-viral, Anti-inflammatory, Antibacterial, Anticancer, Antiviral.

(C) 2020 The Authors. Published by Innovare Academic Sciences Pvt Ltd. This is an open access article under the CC BY license (http://creativecommons. org/licenses/by/4. 0/) DOI: http://dx.doi.org/10.22159/ajpcr.2020.v13i7.37430

\section{INTRODUCTION}

A virus is a biological mediator that reproduces inside the cells of living hosts. When diseased by a virus, a host cell is forced to harvest thousands of undistinguishable copies of the original virus at an extraordinary rate. Unlike most living gears, viruses do not have cells that replicate; novel viruses are assembled in the infected host cell. But unlike still simpler infectious agents, viruses consist of a gene, which gives them the ability to mutate and progress. Over 5000 species of viruses have been revealed [1]

The upbringings of viruses are unclear: Some may have evolved from plasmids, pieces of DNA that can move between cells while others may have evolved from bacteria. A virus consists of two or three parts: Genes, made from either DNA or RNA [2], long molecules that carry genetic material; a protein coat that safeguards the genes; and in some viruses, an envelope of fat that surrounds the protein coat and is used [1,3], in combination with precise receptors, to enter a new host cell. Viruses vary in shape from the modest helical and icosahedral to more complex structures. Viruses range in size from 20 to 300 nanometers; it would take 33,000-500,000 of them, side by side, to stretch to $1 \mathrm{~cm}$ ( $0.39 \mathrm{in})$ viruses [4] blow-out in many habits. Just as many viruses are very unambiguous as to which host species or tissue they attack, each class of virus relies on a particular method for proliferation. Plant viruses are often spread from plant to plant by insects and other creatures, known as vectors. Some viruses of animals, including humans [5], are spread by exposure to infected bodily fluids. Viruses such as influenza are blow-out through the air by droplets of moisture when people cough or sneeze. Viruses such as norovirus are transmitted by the fecal-oral route, which encompasses the contamination of hands, food, and water [6]. Rotavirus is frequently spread by shortest contact with infected children. The human immunodeficiency virus (HIV) is conveyed by body fluids transferred during sex. Others, such as the dengue virus, are spread by blood-sucking insects $[5,7]$.

Viral contagions can cause disease in humans, animals, and even plants. However, they are usually eliminated by the immune system, deliberating lifetime immunity to the host for that virus. Antibiotics have no effects on viruses [3], but antiviral drugs have been established to treat life-threatening infections. Vaccines that produce lifelong immunity can preclude some viral infections [7].

Pyrimidine is a 1,3- (or meta) diazine and may be regarded as being derived from benzene by the replacement of two meta- $\mathrm{CH}$ group by $-\mathrm{N}=$. Pyrimidine is the heterocyclic aromatic compounds containing two nitrogen atoms at positions 1 and 3 of the six-membered rings [1]. Pyrimidine nucleus includes the class of natural and nonnatural products, many of which exhibit different useful biological potentials [2] and clinical applications in the new era. Pyrimidine derivatives have been very well known for their therapeutic applications in the microbial world [3]. The pyrimidine nucleus is present in thiamin, cytosine and uracil. Therefore the pyrimidine nucleus is the building block of DNA and RNA [4]. Substituted pyrimidines possess very widely in living organisms and were some of the first nucleus studied by the organic chemists [5]. The literature review indicated that compounds contain pyrimidine scaffold having biological activities such as anticancer, antiviral, antibacterial, antihypertensive, anticonvulsant, anti-inflammatory, antihistaminic, antithyroid, anti-Alzheimer's, and antibiotics [6] As a result, remarkable pharmacological efficiency of pyrimidine derivatives with intensive research has been focused on antiviral potentials of pyrimidine nucleus [7]

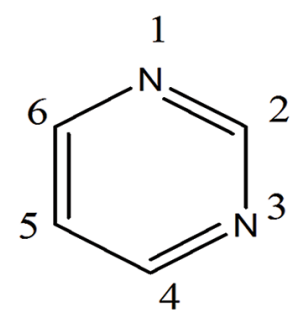




\section{PYRIMIDINE}

The recent pandemic threat posed by the viral pathogens such as coronavirus, and influenza virus (Fig. 1) implies that disease emergence and spread are not limited by geographical boundaries. In many cases, the animals are found to be the source of infection for human infection [1]. Only 87 out of 1399 human pathogens have been first reported in humans in the years since 1980 [4] coronavirus will have a larger negative effect on the global economy than the severe acute respiratory syndrome (SARS) outbreak in 2003. At the time of SARS, China was the sixth-largest economy, accounting for only $4.2 \%$ of in our world gross domestic product (GDP). China is currently the world's second-largest economy, secretarial for $16.3 \%$ of world GDP. Consequently, any slowdown in the Chinese economy sends not ripples but waves crossways the globe, Indian health service said in a commentary on coronavirus outbreak [5].
In this scenario, the coronavirus and resulting measures will reduce global real GDP by 0.4 percent in 2020. On the other hand, if detention measures initiate to lift on February 10, the impact on global GDP will be more limited, subsequent in a $0.1 \%$ reduction in global GDP growth in 2020 and $0.4 \%$ reduction in China's annual growth [6].

New era the different types of new viral strain and viral infections are growing up. Hence, the importance of antiviral drugs is valuable for our country. Heterocyclic moiety like pyrimidine is the basic building block of most of the drugs such as antiviral agents. In our article, we say that we can find drugs for antiviral infection if different changes are made in the pyrimidine nucleus [2].

\section{ANTIVIRAL DRUGS AND THEIR MECHANISM OF ACTION}

All of the currently licensed drugs for systemic therapy of herpesvirus infections share the same target, viral DNA polymerase (Fig. 2). The

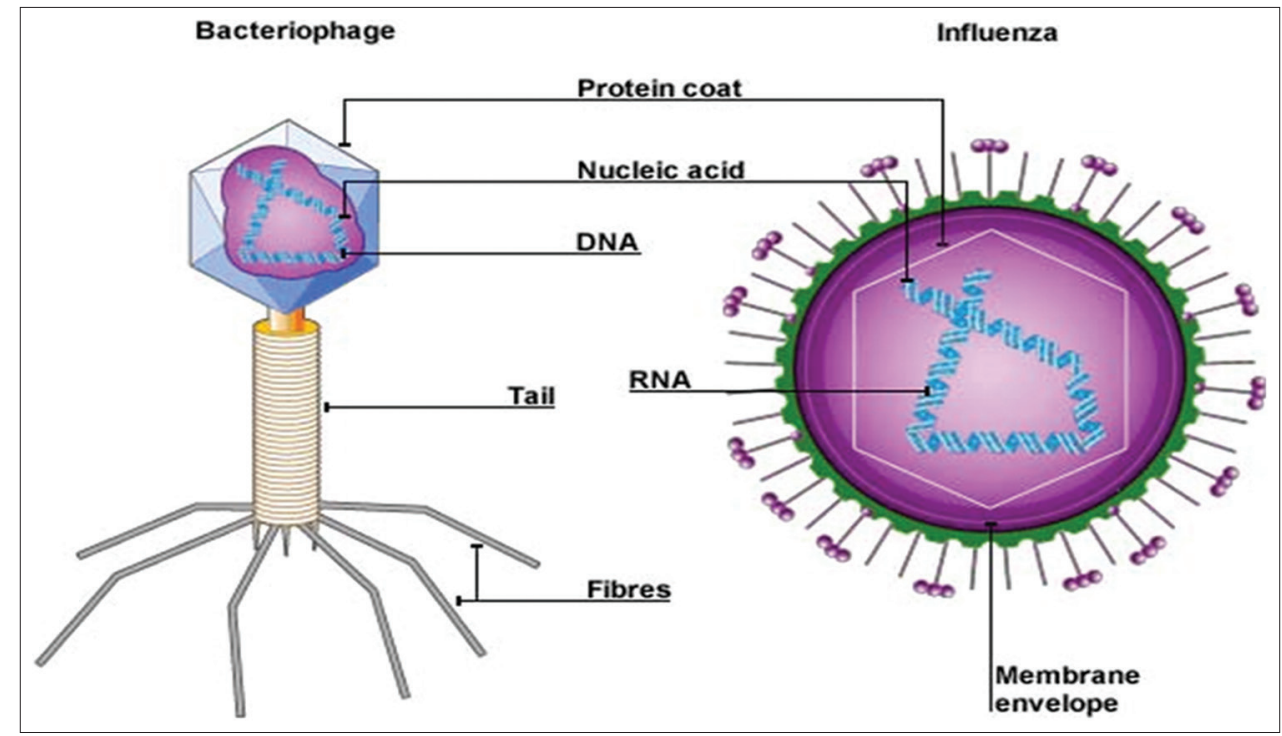

Fig. 1: Structure of virus

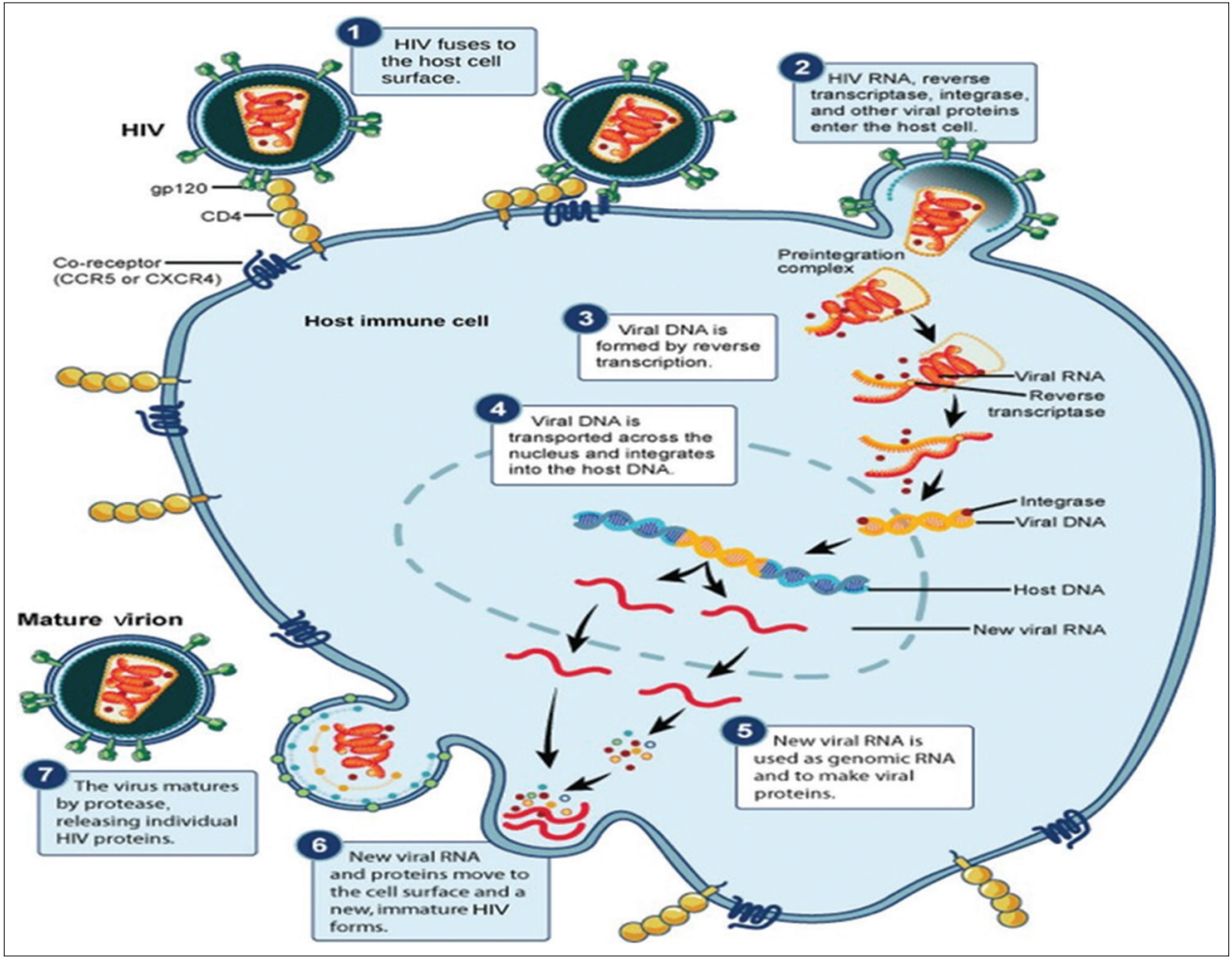

Fig. 2: Mechanism of action of antiviral drugs 
most commonly used drugs are the nucleoside analogs acyclovir and ganciclovir. Acyclovir, its more bioavailable prodrug valacyclovir and famciclovir (the prodrug of penciclovir) are used for herpes simplex virus (HSV) and varicella-zoster virus (VZV) infections but have weak anti-cytomegalovirus (CMV) activity. Ganciclovir and its valine ester prodrug valganciclovir have in vitro activity against HSV, VZV, and CMV and are FDA-approved for CMV infection, where antiviral potency outweighs the increased toxicity as compared with acyclovir [1].

Acyclovir is mono-phosphorylated by thymidine kinase (TK) expressed by HSV (UL23) or VZV (ORF36) and then converted by cellular kinases to the active form, acyclovir triphosphate. Acyclovir triphosphate inhibits HSV and VZV replication by competitive inhibition of viral DNA polymerase and by chain termination of viral DNA strands $[1,2]$. Selectivity is related to preferential activation of acyclovir by viral TK and to the greater sensitivity of viral compared with cellular DNA polymerase to acyclovir triphosphate. Penciclovir, the active metabolite of famciclovir, has a similar mechanism of activation and action. Ganciclovir is monophosphorylated by the CMV UL97 kinase, or HSV, or VZV TK, with subsequent antiviral action analogous to acyclovir. Unlike acyclovir, ganciclovir is not an obligate chain terminator, but rather causes a slowing and subsequent cessation of viral DNA chain elongation [3].

Foscarnet, a pyrophosphate analog, and cidofovir, a nucleotide analog, do not depend on prior activation by viral enzymes. Foscarnet binds selectively to viral DNA polymerase at the pyrophosphatebinding site, blocking of cleavage of the pyrophosphate moiety from deoxynucleotide triphosphates, in turn, halting DNA chain elongation. Cidofovir is phosphorylated by cellular enzymes, and once activated acts as a potent inhibitor of the viral DNA polymerase. Foscarnet and cidofovir are typically used as second- and third-line herpesvirus drugs, respectively, when there is either suspected or documented resistance to initial therapy or dose-limiting toxicities of first-line drugs.

The use of these antiviral drugs may be affected by dose-limiting toxicities. While acyclovir is usually considered relatively nontoxic, high doses are associated with nephrotoxicity [4], and encephalopathy $[5,6]$. High-dose valacyclovir has been associated with thrombotic microangiopathy in immune-compromised hosts [7]. Ganciclovir and valganciclovir frequently cause myelosuppression, especially neutropenia [8,9]. Foscarnet is associated with significant nephrotoxicity and electrolyte abnormalities [10,11]. Cidofovir is associated with nephrotoxicity and neutropenia when administered intravenously [12], and with application site irritation when administered topically [13].

\section{ANTIVIRAL POTENTIALS OF PYRIMIDINE NUCLEUS}

In the present world, extremely active antiretroviral rehabilitation (highly active antiretroviral therapy) is a treatment regimen for HIV infections, concerning the coadministration of nucleoside reverse transcriptase inhibitors (NRTI), non-NRTI, and protease inhibitors. This therapy destroys the replication of HIV and controls disease progression in HIV patients [14]. Due to the emergence of drugresistant HIV variants and adverse effects of the drugs makes, a drastic increase in the number of patients with HIV infection/AIDS has failed to respond to the current antiviral therapy [15]. Therefore, there is a continuous necessity to develop novel anti-HIV drugs that are effective against drug-resistant viruses and produce no adverse effect [16].

Thymidine analog of zidovudine, which consists of azido group is substituted at the $3^{\text {rd }}$ position of the deoxyribose moiety is an act in contradiction of RNA tumor viruses (retroviruses) that are the causative mediators of AIDS and T-cell leukemia [20]. Furthermore, zalcitabine is another useful drug and is given in combination with zidovudine. Another pyrimidine nucleoside analog-like stavudine is initiate to show significant activity against the HIV virus when given in combination with Zidovudine [5].

Pyrimidine derivatives possess very good antiviral properties, such as 5-iododeoxyuridine and 5-iodo-2'deoxyuridine (IDU), have been widely utilized for viral infections [17]. 5-Trifluoromethyl-2'-deoxyuridine has been establishing useful against infections resistant to IDU therapy. The derivatives such as 1-(3-Azido-2, 3-dideoxypentofuranosyl)-5-methyl-2, $4(1 \mathrm{H}, 3 \mathrm{H})$ pyrimidinedione [18] is a leading inhibitor of the in vivo replication and cytopathic movement of HIV and has been accepted for the use for the inhibition of AIDS and severe AIDS-related complex (ARC) [19]. The combination of lamivudine and zidovudine is an effective anti-AIDS; effective cidofovir is used for especially in the treatment of CMV.

Thymidine derivative of zidovudine, which contain azido group is relieved at the $3^{\text {rd }}$ position of the deoxyribose moiety is an act against RNA tumor viruses (retroviruses) that are the causative agents of AIDS and T-cell leukemia [20]. Furthermore, zalcitabine is another useful drug and is given in combination with zidovudine. Another pyrimidine nucleoside analog-like stavudine is initiate to show significant activity against the HIV virus when given in combination with zidovudine [5].

An effective and dependable synthesis of the heterocyclic scaffold-like methyl-3-hydroxy-4-oxo-4H-pyrido [1, 2-a] pyrimidine-2-carboxylate, the scope of the synthesis regarding the introduction of substituent's on the pyrido fused ring is explored. Thus, they conceived a new scaffold for HIV-1 integrase inhibitors in the viral therapy [21].

In 2013, Tichy et al. synthesized two series of new 4-aminopyrimide indole ribonucleoside derivatives. All of these compounds were screened for antiviral activity against HCV and dengue viruses. The compound (1) showed significant anti-dengue and anti-HCV activity but with approximately level of cytotoxicity. The compound (2) exhibited very good anti-HCV activity with low cytotoxicity [22].
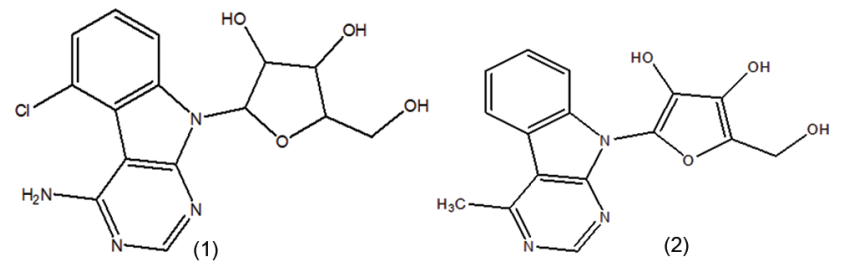

Tremblay et al., 2013, established the synthesis of benzofurano [3, 2-d] pyrimidine -2-one and tested the synthesized compounds for the nucleotide competing HIV-1 reverse transcriptase inhibitors (NcRTI) activity. The compound (3) showed auspicious overall in vitro properties as HIV-1 RT reverse transcriptase inhibitors [23]. The compounds expose the potential of benzofurano $[3,2$-d] pyrimidine-2-one derivatives therefore they included as a class of future anti-HIV agent [5].

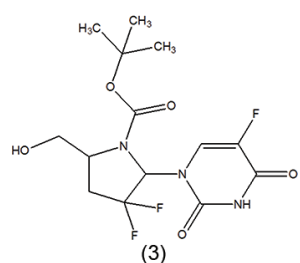

In 2008, Rashed et al. disclosed synthesis and antiviral evaluation of some new pyrimidine analogues such as pyrazole and fused pyrazolopyrimidine derivatives (4). Antiviral assay was performed, and using different concentration of the series of compounds synthesized which consequently exposed their anti-HCV potential. S-acyclic nucleoside derivatives at $20 \mu \mathrm{g} / 150$ cells exhibited slighter potency than fused pyrazolo $[3,4]$ pyrimidine [16].

In 2013, Rostamized et.al. generated an elaborated and antiviral activity of compounds such as pyrazolo [3, 4-d] pyrimidine derivatives. They deliberated the activity on Enterococcus raffinosus and staphylococcus aurous. The pyrazolo [3, 4] pyrimidines having different substitutions and their minimum inhibitory concentration are enlished [24]. 
<smiles>[Y]C1NC=Nc2c1cnn2C</smiles>

(4)

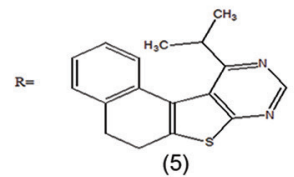

(5)
$\mathrm{X}=\mathrm{O}, \mathrm{Cl}, \mathrm{S}$,<smiles>COC(COC(C)C)OCCO</smiles>

Kim et al., 2013, identified a series of triazolothieno pyrimidine (TTPM) analogs as potent HIV-1 replication inhibitors by performing a cellbased full replication assay, it was established that aryl substituted TTPM derivatives (5), (6), and (7) exhibited potent inhibitory activity scaffold with acceptable well-being margins [16].

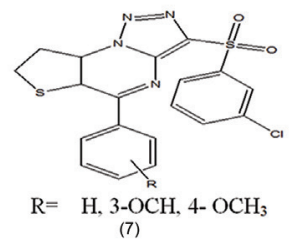

Sakakibara et al., 2013, generated a library of compounds such as 3-(3,5dimethylbenzyl) uracil scaffold and screened them for nonnucleoside HIV-1 reverse transcriptase inhibition potential [25]. Among these compounds, (8) was establish to be potent against HIV-1 activity (EC50 $=0.03 \mu \mathrm{M}$ and a high selectivity index $=2863$ ). Thus, the compound may serve as a prime for further optimization of anti-HIV drugs [16].

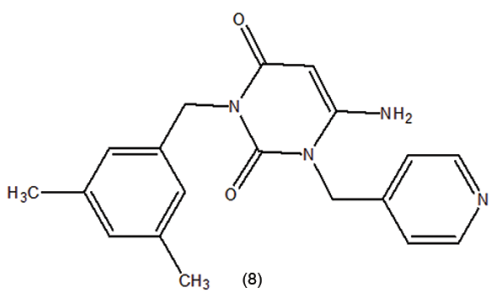

Two series of new 4-aminopyrimido [4,5-b]indole ribonucleoside derivatives were synthesized and different biological activity was tested by Tichy et al., 2013. All of these analogs were tested for antiviral activities against HCV and dengue viruses. Compound (9) showed potent anti-dengue and anti-HCV activity but with some level of cytotoxicity. In the case of compound (10) exhibited high anti-HCV activity with low cytotoxicity [16].<smiles>Nc1ncnc2c1c1c(Cl)cccc1n2C1OC(CO)C(O)C1O</smiles><smiles>Nc1ncnc2c1c1ccccc1n2C1OC(CO)C(O)C1O</smiles>

Mohamed et al., 2010, some substituted that pyrimidine derivatives were designed, synthesized, and evaluated for their anti-HSV-1 potential [26]. The antiviral evaluation exhibited that the compounds (11) and (12) have over $90 \%$ potent inhibition comparable to standard Acyclovir [21]. These compounds may be considered highly promising antiviral agent for further examinations [16]<smiles>COc1ccc([C@H]2NC(SC(C(C)=O)C(C)=O)=N[C@H](c3ccc4c(c3)CCCC4)C2=O)cc1OC</smiles><smiles>COc1ccc([C@H]2C(=O)[C@@H](c3ccc4c(c3)CCCC4)N=C3SC(C)C(=O)N32)cc1OC</smiles>

Retrovir (AZT-16, 34) is a potent inhibitor of the in vivo replication and cytopathic effects of HIV and has been recently accepted for the use against AIDS and severe ARC [31]. At present acyclovir is the only medicine for genital herpes. The oral formulation of acyclovir is effective against both first- and second-degree recurrence-genital herpes with minimal side effects. Ganciclovir has exhibited respectable in vivo inhibitory potential against HCV1 and HCV2 [27]. Several members of a series of acyclic nucleosides, which contain a fused pyrimidine ring (mainly purine), are established to be effective antivirals [9]. Famciclovir and valacyclovir are drugs used for several DNA viruses, including HSV types 1 and 2, VZV, and Epstein-Barr virus [27].

Penciclovir is a powerful medication useful for the treatment of topical recurrent herpes, libialis. Cidofovir is an antimetabolite for deoxycytidine triphosphate which is used for the treatment of CMV in AIDS patients. Lamivudine is an operative anti-AIDS drug when used in combination with zidovudine [28]. Thymidine analogs of zidovudine is a compound which contains azido group is substituted at the 3-position of the deoxyribose moiety. It is mainly active against RNA tumor viruses (retroviruses) that are the causative agents of AIDS and T-cell leukemia. It is used in the treatment of AIDS and ARC to control opportunistic infections by raising absolute $\mathrm{CD}^{+}$lymphocyte counts. Furthermore, zalcitabine is a useful another alternative drug to zidovudine [16]. It is given in combination with zidovudine, when $\mathrm{CD}^{+}$cell counts fall below 300 cells $/ \mathrm{mm}^{3}$ [5]. Didanosine is a purine deoxynucleoside, which is a scaffold of inosine. Didanosine acts against HIV RT and exerts an antiviral effect on the retroviruses. Combined with zidovudine, antiretroviral activity of didanosine is augmented [29].

Mizuhara et al., 2012, identified the synthesis of pyrimidine [1, 2-c] $[1,3]$ benzothiazin-6-imine analog as a potent antiretroviral agent. Among them, compounds (13) and (14) exhibited threefold higher antiHIV activity than that of PD 404182 [16].<smiles>COc1ccc(-c2ccc3c(c2)SC(=N)N2CCCN=C32)cc1</smiles><smiles>N=C1Sc2cc(-c3ccc4c(c3)OCO4)ccc2C2=NCCCN12</smiles>

Rai et al., 2013, designed, synthesized, and screened 3benzyloxylinked pyrimidine phenylamine derivatives for their in vitro anti-HIV potentials in MT-4 cell cultures. Virtually, all the derivatives exhibited inhibitory potentials of HIGH SELECTIVITY INDEX. The compounds such as (15) and (16) exhibited outstanding antiretroviral activity against wt HIV-1 with low cytotoxicity $(\mathrm{EC} 50=0.07 \mu \mathrm{M}, \mathrm{CC} 50>347$ $\mu \mathrm{M}, \mathrm{SI}>4870 ; \mathrm{EC} 50=0.05 \mu \mathrm{M}, \mathrm{CC} 50=42 \mu \mathrm{M}, \mathrm{SI}=777$, respectively), associated to marked medication, nevirapine $($ EC50 $=0.113 \mu \mathrm{M}$, CC50>15 $\mu \mathrm{M}$, SI $>133$ ) ASDAr [16]. 


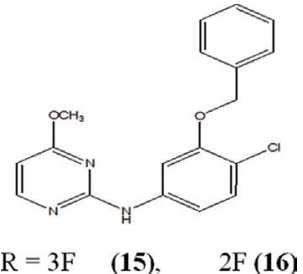

After the intra cellular conversion of drug like stavudine can act as more potent anti-HIV activity. It is additional effective than zidovudine or didanosine for treatment in patients for delaying the development of HIV infection. Therefore, it is recommended for patients with advanced HIV infection [30].

20,30-dideoxy-20,20-difluoro-40-aza nucleoside derivatives of both pyrimidine and purine nucleobases were synthesized by Martínez-Montero et al., 2012, and screened them for their anti-HIV1 and anti-HCV potentials. Among the series, 40-azanucleosides were originated to be the most active compound (EC50 $=36.9 \mu \mathrm{M})$ and none of the derivatives were found to possess anti-HCV activity [16].

\section{CONCLUSION}

We believe that, pyrimidine nucleus has an important value in our current scenario. We know that due to the globalization the mankind generation having numerous development or different changes from our old generation as well as our health problems too has increased. Thus, it is an important and an urgent paradigm to change in pyrimidine scaffold to improve powerful resistive medicines to our new generation to build people to keep their health in a good manner particularly against the upcoming viruses due to the inner nucleic acids also contain the base unit of pyrimidine scaffold. The literature reviews revealed that the pyrimidines are considered as a versatile scaffold for antiviral therapy.

\section{ACKNOWLEDGMENT}

Declared none.

\section{AUTHOR'S CONTRIBUTION}

All authors have contributed equally for bringing this review article effectively.

\section{CONFLICTS OF INTEREST}

The author(s) confirm that this article content has no conflicts of interest.

\section{REFERENCES}

1. Wagstaff AJ, Faulds D, Goa KL. Aciclovir. A reappraisal of its antiviral activity, pharmacokinetic properties and therapeutic efficacy. Drugs 1994;47:153-205.

2. Elion GB. Acyclovir: Discovery, mechanism of action, and selectivity. J Med Virol 1993;1:2-6.

3. Gilbert C, Bestman-Smith J, Boivin G. Resistance of herpesviruses to antiviral drugs: Clinical impacts and molecular mechanisms. Drug Resist Updat 2002;5:88-114.

4. Wagstaff AJ, Faulds RA, Weiss P, Ritz RF. Acyclovir-induced neurotoxicity: Concentration-side effect relationship in acyclovir overdose. Am J Med 1993;94:212-5

5. Elion GB, Furman PA, Fyfe JA, Miranda P, Beauchamp L, Schaeffer HJ. Selectivity of action of an antiherpetic agent, 9-(2-hydroxyethoxymethyl) guanine. Proc Natl Acad Sci USA 1977;74:5716-20.

6. Lowance D, Neumayer HH, Legendre CM, Squifflet JP, Kovarik J, Brennan PJ, et al. Valacyclovir for the prevention of cytomegalovirus disease after renal transplantation. International valacyclovir cytomegalovirus prophylaxis transplantation study group. N Engl J Med 1999;340:1462-70.

7. Ormrod D, Scott LJ, Perry CM. Valaciclovir: A review of its long term utility in the management of genital herpes simplex virus and cytomegalovirus infections. Drugs 2000;59:839-63

8. Kalil AC, Freifeld AG, Lyden ER, Stoner JA. Valganciclovir for cytomegalovirus prevention in solid organ transplant patients: An evidence-based reassessment of safety and efficacy. PLoS One 2009; $4: \mathrm{e} 5512$

9. Crumpacker CS. Ganciclovir. N Engl J Med 1996;335:721-9.

10. Wagstaff AJ, Bryson HM. Foscarnet. A reappraisal of its antiviral activity, pharmacokinetic properties and therapeutic use in immunocompromised patients with viral infections. Drugs 1994;48:199-226.

11. Deray G, Martinez F, Katlama C, Levaltier B, Beaufils H, Danis M, et al. Foscarnet nephrotoxicity: Mechanism, incidence and prevention. Am J Nephrol 1989;9:316-21.

12. Safrin S, Cherrington J, Jaffe HS. Clinical uses of cidofovir. Rev Med Virol 1997; 7:145-56

13. Lagoja IM. Pyrimidine as constituent of natural biologically active compounds. Chem Biodivers 2005;2:1-50.

14. Ghomi AS, Ali M. An efficient route to the synthesis of pyrimidine2-ones under ultrasound irradiation. Dig J Nanomater Biostruct 2010;5:303-6.

15. Dansena H, Dhongade HJ, Chandrakar K. Pharmacological potentials of pyrimidine derivatives: A review. Asian J Pharm Clin Res 2015;8:171-7.

16. Amir M, Javed SA, Kumar H. Pyrimidine as anti-inflammatory agent: A review. Indian J Pharm Sci 2007;69:337-43.

17. Sharma V, Chitranshi N, Agarwal AK. Significance and biological importance of pyrimidine in the microbial world. Int J Med Chem 2014;2014:202784.

18. Wesley F, Nadia M. Synthesis and molecular docking studies on three novel dihydropyrimidine derivatives. J Mol Struct 2019;1192:274-287.

19. Nongkhlaw RL, Tumtin S. Recent progress in the chemistry of dihydropyrimidinones. Rasayan J Chem 2009;3:662-76.

20. Shmalenyuk ER, Chernousova LN, Karpenko IL, Kochetkov SN. Inhibition of Mycobacterium tuberculosis strains H37Rv and MDR MS-115 by a new set of C 5 modified pyrimidine nucleosides. Bioorg Med Chem 2013;21:487-4.

21. Guo D, Zhang X, Wang R, Zhou Y, Li Z, Xu J. Structural modifications of 5, 6-dihydroxypyrimidines with anti-HIV activity. Bioorg Med Chem Lett 2012;22:7114-8.

22. Shahu M, Siddiqie N. A review on biological importance of pyrimidines in the new era. Int J Pharm Pharm Sci 2016;5:8-21.

23. Brujce PY. Organic Chemistry. $3^{\text {rd }}$ ed. Singapore: Pearson Education; 2007.

24. Sharma P, Rane N, Gurram VK. Synthesis and QSAR studies of pyrimido $[4,5$-d]pyrimidine-2, 5-dione derivatives as potential antimicrobial agents. Bioorg Med Chem Lett 2004;14:4185-90.

25. de Clercq E. Antiviral drugs in current clinical use. J Clin Virol 2004;30:115-33

26. Kapoor VK, Singh H. In: Jain MK, editor. Medicinal and Pharmaceutical Chemistry. Vol. 8. Delhi, India. Vallabh Prakashan; 2005.

27. Sacchi A, Laneri S, Rossi FB. The molecular structures of the isomeric compounds 5, 7-dimethoxyimidazo [1,2-c]pyrimidine and 7-methoxy1-methylimidazo[1, 2-a] pyrimidine 5(1H)-one. Eur J Med Chem 1997;32:677.

28. Gupta JK, Chaudhary A, Dudhe R, Varuna K, Sharma PK, Verma PK. A review on the synthesis and therapeutic potential of pyrimidine derivatives. Int J Pharm Sci Res 2010;1:34-44.

29. Vaidya VP, Mathias P. Synthesis and pharmacological evaluation of some naptho [2, 1-b] furo [3, 2-b] pyrimidines. Indian J Heterocycl Chem 2005;14:189-92.

30. Chauhan M, Kumar R. Medicinal attributes of pyrazolo [3, 4-d] pyrimidines: A review. Bioorg Med Chem 2013;21:5657-68.

31. Sakakibara N, Hamasaki T, Baba M. Synthesis and evaluation of novel 3-(3, 5-dimethyl benzy) uracil analogues as potential anti HIV-1 agents. Bioorg Med Chem 2013;21:5900-6.

32. Shook BC, Chakravarty D, Barbay JK, Wang A, Leonard K, Alford V, et al. Substituted thieno [2, 3-d] pyrimidines as adenosine A2A receptor antagonists. Bioorg Med Chem Lett 2013;23:2688-91.

33. Kaspersen SJ, Sundby E, Charnock C, Hoff BH. Activity of 6-arylpyrazolo [2, 3-d] pyrimidine-4-amines to Tetrahymena. Bioorg Chem 2012;44:35-41.

34. Mansuri MM, Martin JC. Chapter 17. Antiviral agents. Annu Rep Med Chem 1987;22:147.

35. Singh P, Kumar R, Sharma BK. Quantitative structure-activity relationship study of 5-iodo-and diaryl-analogues of tubercidin: Inhibitors of adenosine kinase. J Enzyme Inhib Med Chem 2003; 18:395-402.

36. Sondhi SM, Goyal RN, Lhoti AM, Shukla SN. Synthesis and biological evaluation of 2-thiopyrimidine derivatives. Bioorg Med Chem Lett 2005; 13:3185-95. 